\title{
A Safe Method for Middle Colic Dissection and Ligation at its Origin in a Laparoscopic Extended Right Hemicolectomy
}

\author{
Frederick H. Koh, MBBS, MRCSEd ${ }^{1}$ and Ker-Kan Tan, MBBS, FRCSEd ${ }^{1,2}$ \\ ${ }^{1}$ Division of Colorectal Surgery, University Surgical Cluster, National University Hospital, National University Health \\ Systems, Singapore, Singapore; ${ }^{2}$ Department of Surgery, Yong Loo Lin School of Medicine, National University of \\ Singapore, Singapore, Singapore
}

\begin{abstract}
Background. Laparoscopic extended right hemicolectomy is regarded as one of the more difficult procedures in colorectal surgery due to the complexity of the dissection around the pancreatic neck to identify the origin of the middle colic artery. ${ }^{1}$ Proper identification and ligation of the middle colic artery at its origin is paramount to achieve complete mesocolic excision. ${ }^{2,3}$ We describe our technique of middle colic vessels dissection in a laparoscopic extended right hemicolectomy.

Methods. Our patient was a 58-year-old female with a stenosing transverse colon adenocarcinoma. The video highlights the key steps of a laparoscopic extended right hemicolectomy with special attention to the dissection and identification of the origin of the middle colic vessels at the pancreatic neck. We utilized a posterior-to-anterior approach for the dissection around the superior mesenteric pedicle.

Results. By carefully skeletonizing the pancreas from the body to the neck, the superior mesentery pedicle is isolated and skeletonized to identify the origin of the middle colic vessels. A posterior-to-anterior approach is used to complete the skeletonisation before ligation of the middle colic vessels at its origin. Operative time was $288 \mathrm{~min}$ with an
\end{abstract}

Electronic supplementary material The online version of this article (doi:10.1245/s10434-016-5507-6) contains supplementary material, which is available to authorized users.

(C) Society of Surgical Oncology 2016

First Received: 26 June 2016;

Published Online: 22 August 2016

K.-K. Tan, MBBS, FRCSEd

e-mail: surtkk@nus.edu.sg estimated blood loss of $40 \mathrm{ml}$. The patient recovered well without complications of pancreatitis and was discharged on postoperative day 5 . Histology revealed a $4-\mathrm{cm}$ moderately differentiated adenocarcinoma with 10 of 34 lymph nodes involved-pT3N2b.

Conclusions. With the increasing popularity of laparoscopic surgery, meticulous laparoscopic dissection of the middle colic vessels is feasible and safe and may potentially help to optimize oncological outcomes for laparoscopic extended right hemicolectomy.

DISCLOSURE None

\section{REFERENCES}

1. Chew SSB, Adams WJ. Laparoscopic hand-assisted extended right hemicolectomy for cancer management. Surg Endosc. 2007;21(9):1654-6.

2. Li Yong. Laparoscopic extended right hemicolectomy with D3 lymphadenectomy. Ann Transl Med. 2015;3(9):124.

3. Lux P, Weber K, Hohenberger W. Laparoscopic surgery for colon cancer: quality requirements for (extended) right hemicolectomy. Chirurg. 2014;85(7):593-8. 